\title{
INFLUENCE OF THE TEXTURE ON MECHANICAL PROPERTIES OF THE Al-Li ALLOY 1441
}

\author{
V.F. SHAMRAY ${ }^{a} *$, A.A. BABAREKO ${ }^{a}$ and \\ O.A. SETJUCOV ${ }^{\mathrm{b}}$ \\ ${ }^{\text {a }}$ A.A. Baikov Metallurgy Institute of Russian Academy, \\ Leninskii prospect 49, 117911 Moscow, Russia; ${ }^{\mathrm{b}}$ All-Russian \\ Institute of Aviation Materials, Moscow, Russia
}

(Received in final form 28 September 1997)

Results of texture investigations in sheet and plate of the $\mathrm{Al}-\mathrm{Li}$ alloy 1441 are presented. The peculiarities of fracture surface morphology formation during fatigue crack propagation tests in dependence on texture inhomogeneity are considered. The deviation of the fatigue crack from the principal direction in center-notched specimens could be related to occurrence of a $\{110\}\langle 112\rangle$ texture. The formation of crystallographic faceted fracture surfaces results from cracks extending by slip along equally shear stressed $\{111\}\langle 110\rangle$ slip systems ahead of the crack tip.

Keywords: Al-Li alloys; Texture; Microcrack; Fracture; Surface; Crystallography

\section{INTRODUCTION}

As already reported earlier (Fridlyander et al., 1994; Rao and Ritchie, 1992; Wu et al., 1994), many peculiarities of the mechanical properties of $\mathrm{Al}-\mathrm{Li}$ alloys can be related to the existence of a strong crystallographic texture. In $\mathrm{Al}-\mathrm{Li}$ alloy semi-products it was observed the lower yield stress value in specimens oriented at angles 0 or $60^{\circ}$ to the rolling direction (the inverse and $60^{\circ}$ anisotropy, Fridlyander et al., 1994). Textured plates of the $\mathrm{Al}-\mathrm{Li}$ alloys 1441 and 8090 show a fatigue crack

* Corresponding author. 
deviation and through-thickness delamination during fracture toughness testing (Rao and Ritchie, 1992; Wu et al., 1994; Roven et al., 1990). The fatigue crack behavior of these materials, which is crystallographic in its character, is determined by localized slip caused by the texture. As a result fracture with pronounced crystallographic shear facets on the fracture surface is initiated. Inhomogeneous localized deformation is favored by the presence of $\delta^{\prime}$-particles (Rao and Ritchie, 1992; Wu et al., 1994). The shearable nature of these $\delta^{\prime}$-precipitates enhances inhomogeneous localized slip deformation and stimulates the crack deviation and tortuous fatigue crack propagation. On the other hand, an enhanced crack propagation resistance and increased fracture toughness of $\mathrm{Al}-\mathrm{Li}$ materials could be associated with slip band crack growth which promotes the crack tip shielding effect.

The problem of fatigue crack propagation and tortuous crack path morphology in $\mathrm{Al}-\mathrm{Li}$ alloys is not properly understood so far since it follows from the above discussion that the deformation texture as well as distinctive features of the $\delta^{\prime}$-precipitates could play an important role in these processes, this paper gives the results of a texture investigation of 1441 alloy plate and sheet in which these problems occur.

\section{EXPERIMENTAL}

The material studied in the present investigation was the $\mathrm{Al}-\mathrm{Li}$ alloy 1441 which was obtained in the form of both $25 \mathrm{~mm}$ thick plate, 1 and $2 \mathrm{~mm}$ thick cold rolled and $7 \mathrm{~mm}$ thick hot rolled sheet. The composition of the alloy is: $1.9 \mathrm{Li}, 1.05 \mathrm{Mg}, 1.73 \mathrm{Cu}, 0.08 \mathrm{Zr}, 0.08 \mathrm{Mn}$ wt pet; the final thermal treatment consisted of water quenching and aging for $24 \mathrm{~h}$ at $150^{\circ} \mathrm{C}$.

Direct pole figures and the integral function $P_{\beta=0-360^{\circ}}(h k l)=f(\alpha)$ for $\alpha$ angles in the interval from 0 to $75^{\circ}$ was measured by means of the automatic texture goniometer DART-UM1 using $\mathrm{CuK}_{\alpha} \mathrm{X}$-radiation. The matrix texture was analyzed by recording (220) and (400) pole figures and the $\delta^{\prime}$-phase texture by recording (100) and (110) pole figures. Through-thickness texture inhomogeneities in the short transverse direction were investigated by means of layer-by-layer texture analysis.

To analyze fatigue crack propagation, standard $5 \mathrm{~mm}$ center-notched fatigue specimens (K20) were taken from the central part of $25 \mathrm{~mm}$ plate 
and the $7 \mathrm{~mm}$ sheet. The tests were performed at a frequency of $5 \mathrm{~Hz}$ in air under $\Delta K$-increasing condition. Compact specimens for eccentric tension test were cut from the $25 \mathrm{~mm}$ plate.

Microstructure and fracture surface relief were examined optically and with the help of an optic goniometer.

\section{RESULTS}

The fracture surface relief depends on the direction of the applied stress and the type of the material. The fracture surface morphology is quite different for the $5 \mathrm{~mm}$ thick LT and TL center-notched specimens which were cut from the central part of $25 \mathrm{~mm}$ plate. In the LT specimens the crack path is deviated from the plane of maximum tensile stress by not more than $5^{\circ}$. The relief of fracture surface is rough in its character and has a saw type profile. In the TL specimens the crack propagation path takes the form of a zig-zag line with individual sections of the path of lengths up to several $\mathrm{cm}$ that are deflected with respect to the notch direction by angles of $53-58^{\circ}$, Fig. 1 . The crack path can change its growth direction to the direction which is mirror-plane symmetric with respect to the plane parallel to the loading direction. As a result wedgelike sections along the crack growth direction with apex angles of $70^{\circ}$ are induced. The fracture surface has a fractal structure consisting of intersecting facets forming diheders elongated in rolling direction with dihedral angles of $70^{\circ}$.

The fracture surface of the compact specimen after eccentric tension test is delaminated on 4-5 layers containing many sublayers inside each of them.

Texture inhomogeneities across the thickness are a characteristic feature of 1441 plate and sheet. In the central layers of these materials the brass-type texture $\{110\}\langle 112\rangle$ with incomplete axial component $\{110\}-\{531\}\langle 112\rangle$ or $\{110\}-\{311\}\langle 112\rangle$ (corresponding to rotation angles around the rolling direction from $19^{\circ}$ to $\left.32^{\circ}\right)$ is developed. In the center of 2 and $1 \mathrm{~mm}$ sheets the $\{123\}\langle 541\rangle$ component is observed. The similar component $\{123\}\langle 634\rangle$ earlier has been attributed to the occurrence of recrystallization in Al-Li alloys (Zeng et al., 1994).

Thus, in the central part of the $25 \mathrm{~mm}$ plate the brass texture $\{110\}\langle 112\rangle$ dominates (Fig. 2). The (004) pole figure of the TL specimen 


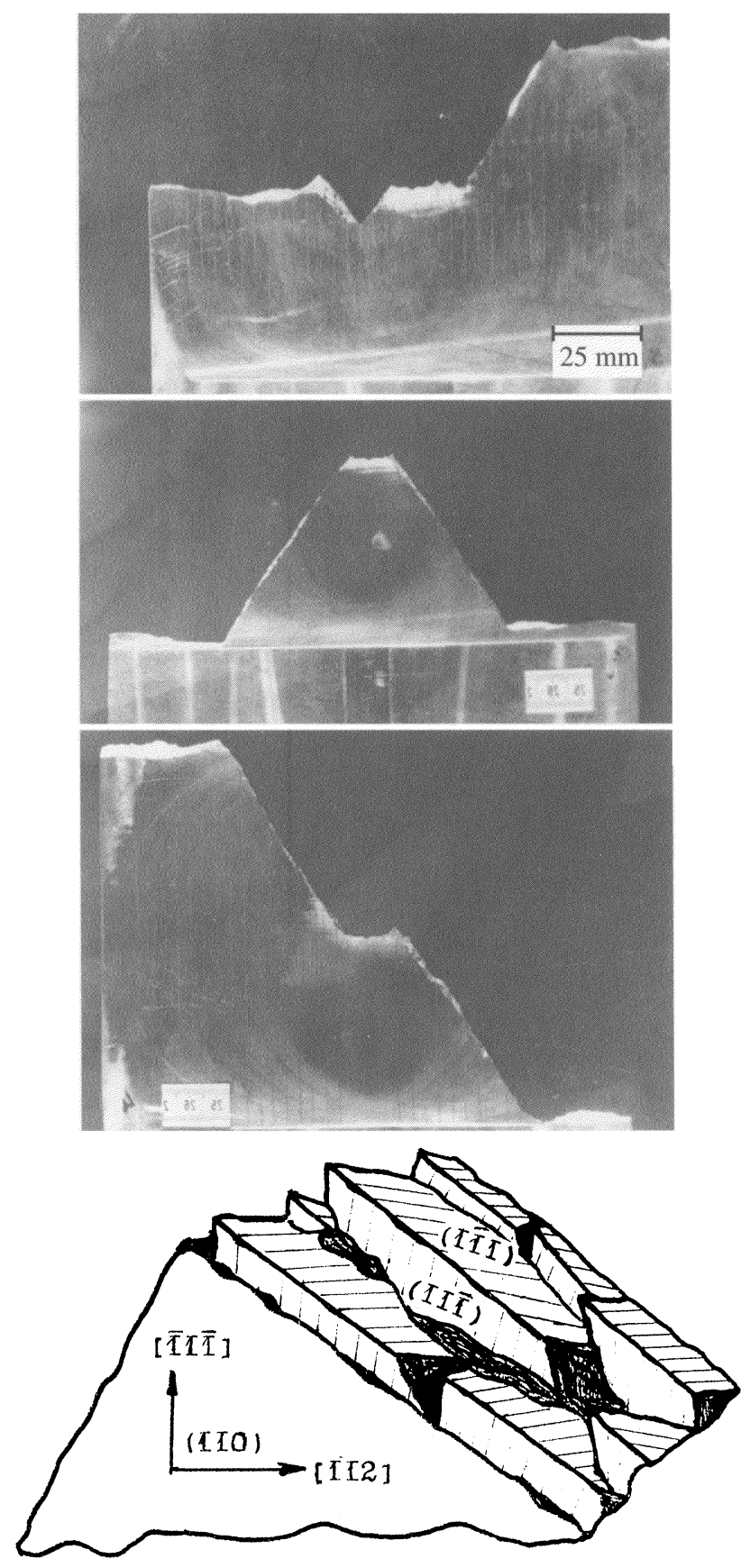

FIGURE 1 The fatigue crack propagation character in TL $5 \mathrm{~mm}$ center-notched specimens from the central part of the $25 \mathrm{~mm}$ plate. In lower part the fine structure fragments of the fracture surface are shown schematically. 

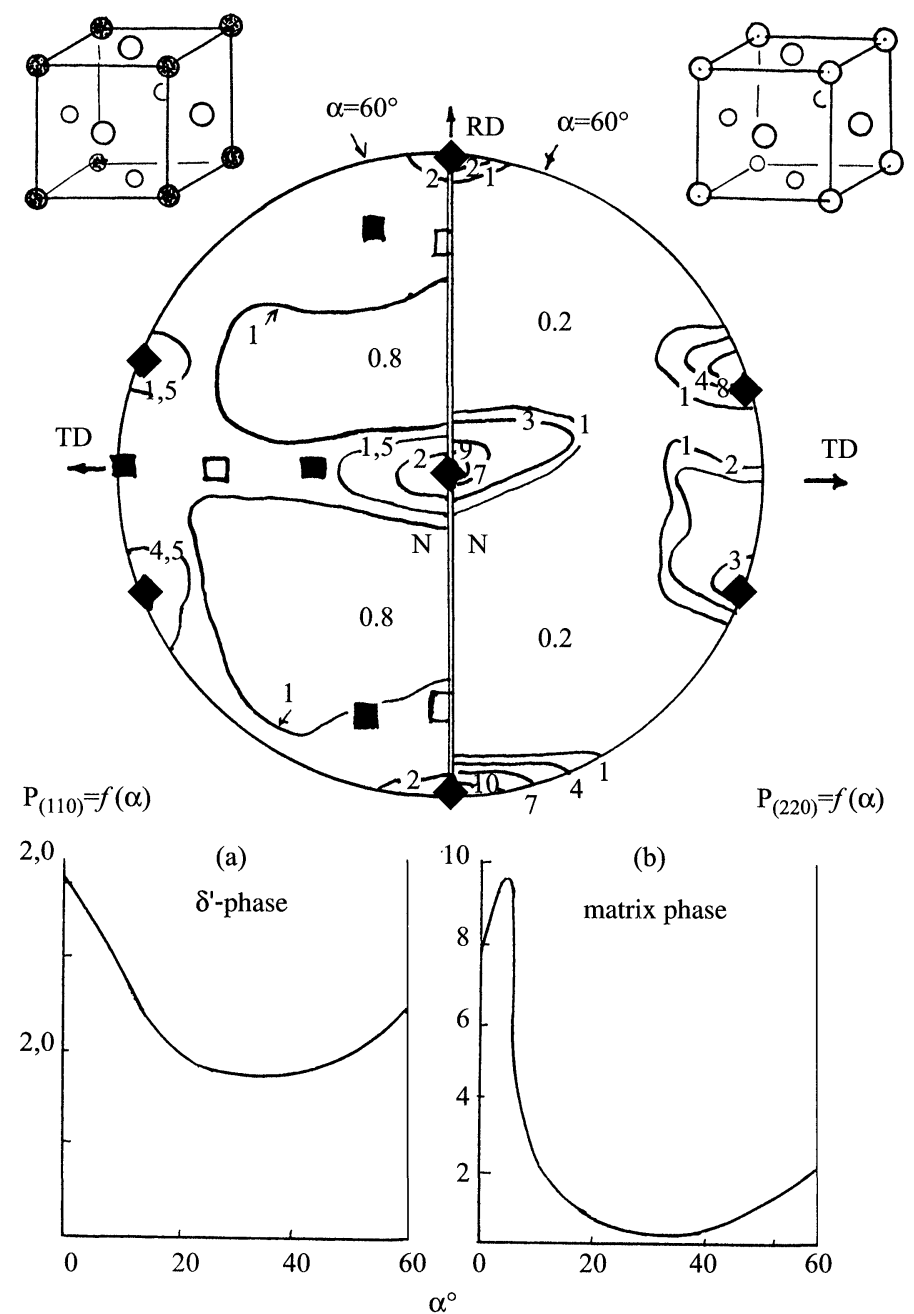

FIGURE $2(110)$ and (220) pole figures and $P_{(\mathrm{hh} 0)}=f(\alpha)$ registered for $5 \mathrm{~mm}$ specimens from central part of the $25 \mathrm{~mm}$ plate ((a) $\delta^{\prime}$ and (b) matrix phases); $\{110\}\langle 112\rangle, \square-(001)[110], \mathbf{\square}-\{310\}\langle 001\rangle$ orientations; $\mathrm{N}$ - short and TD - long transverse directions, RD - rolling direction. In upper part the unit cells of $\delta^{\prime}$ and matrix phases are shown. 
fracture surface, macroscopically plane and deflected by $55^{\circ}$ from the

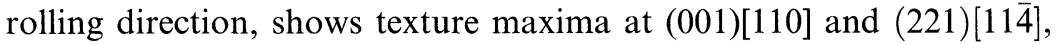
(Fig. 3). Under consideration of the original specimen setting during the experiment, these maxima correspond to the two mirror-plane symmetric components (110)[112] and (110)[11̄2] of the brass texture.

In the surface regions of sheet and plate the shear texture components (001)[110] and (111)[110] arise. The first orientation is accompanied by $(113)[33 \overline{2}]$ and $(112)[11 \overline{1}]$ orientations which are related to $(001)[110]$

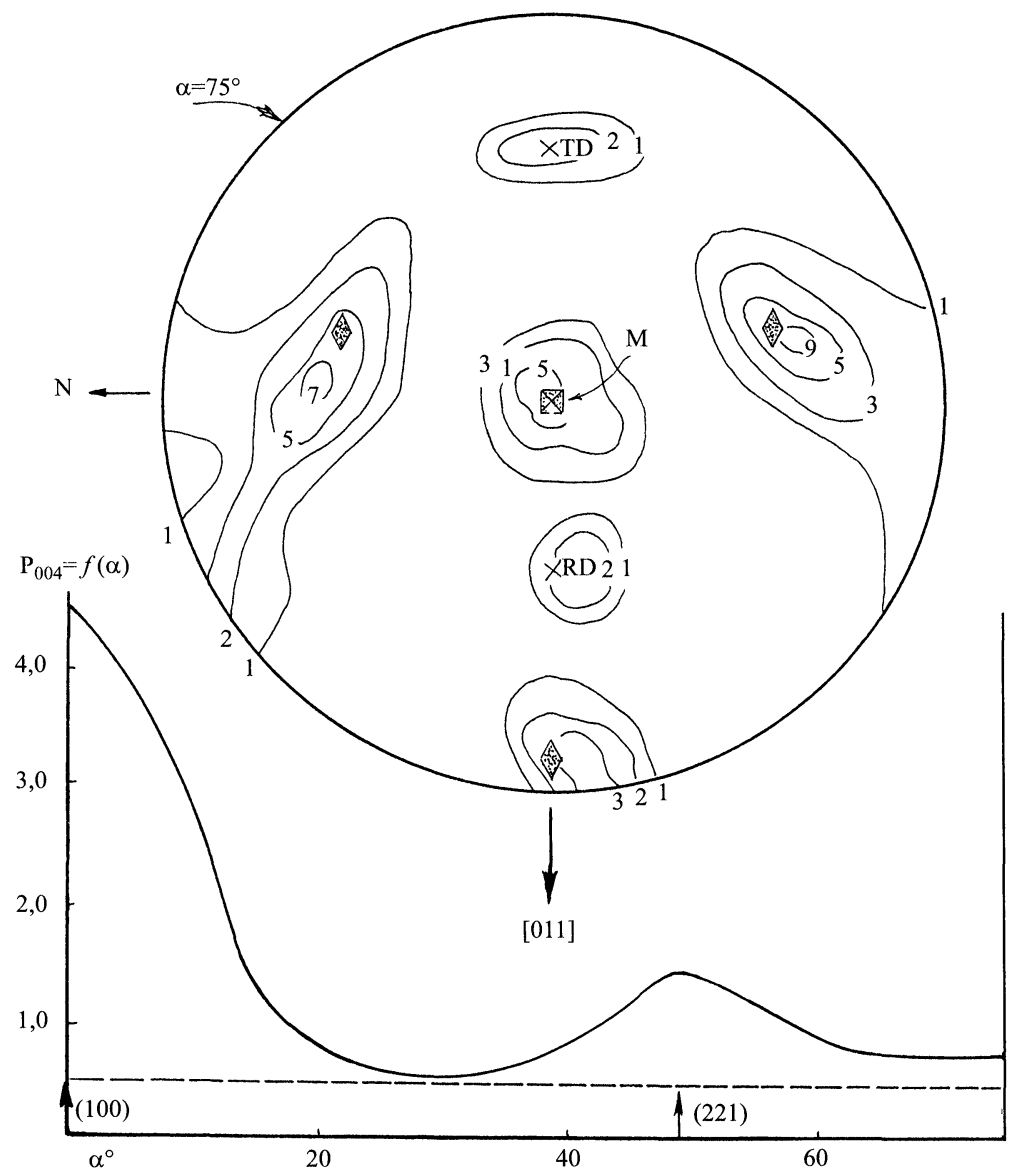

FIGURE 3 (004) pole figure and $P_{(004)}=f(\alpha)$ registered from the fracture surface of TL $5 \mathrm{~mm}$ specimen from central part of the $25 \mathrm{~mm}$. (M: fracture surface, RD and TD: rolling and long transverse directions). The orientations: $-(211)[11 \overline{4}],-(100)$. 
by rotation around the transverse direction [110] by $25^{\circ}$ and $30^{\circ}$ respectively. Formation of these two components is assumed to be the result of the further shear deformation of (001)[110] textured material. The shear induced components penetrate up to 0.25 of sheet or plate thickness. In the $2 \mathrm{~mm}$ thick sheet, the intensity of the shear induced $\{001\}\langle 110\rangle$ component reaches a maximum in a near-surface region spaced at 0.125 of sheet thickness.

The texture of the $\delta^{\prime}$-phase in 1441 sheet and plate is generally similar to the matrix texture (Fig. 4). However, in the pole figure of the $\delta^{\prime}$-phase in the specimen taken from the central part of the $25 \mathrm{~mm}$ plate the additional components $\{100\}\langle 001\rangle$ and $\{310\}\langle 001\rangle$ could be seen (Fig. 2(a)).

The grains of $25 \mathrm{~mm}$ plate are disc shaped and are elongated in the rolling direction. There are large grain size variations in the structure. In the central part of the plate the grains are typically $100 \mu \mathrm{m}$ thick, $500 \mu \mathrm{m}$ wide and $\sim 5 \mathrm{~mm}$ in length, but larger grains up to $0.3 \times 2 \times 10 \mathrm{~mm}$ also could be observed.

Figure 5 presents the stereographic projection of a $(110)[112]$ textured f.c.c. material with the traces of the primary octahedral slip-plane and
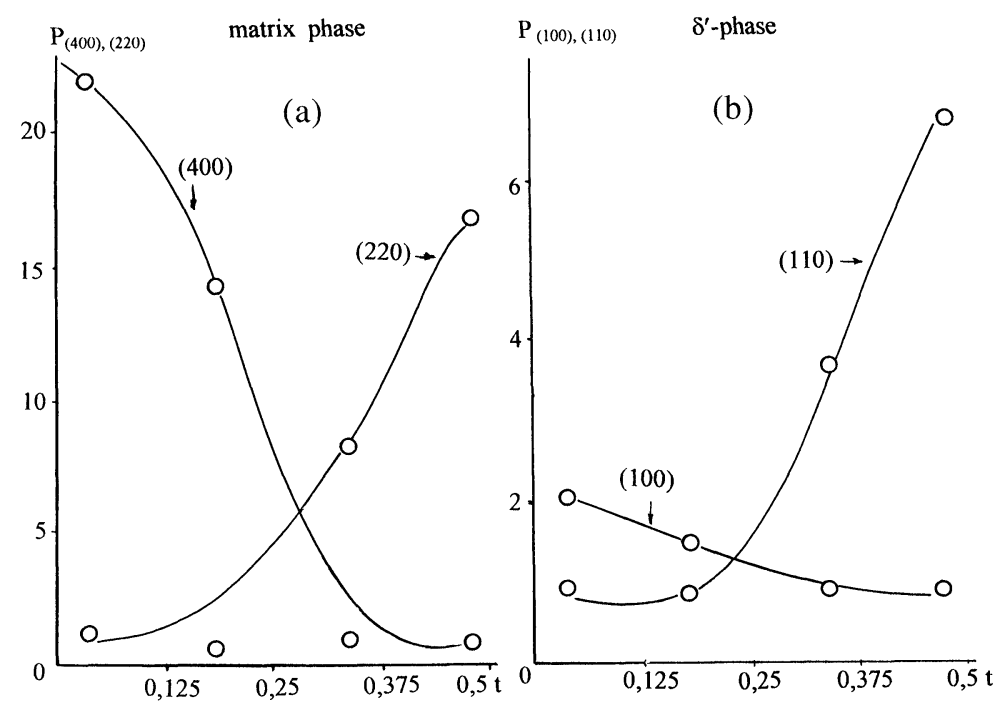

FIGURE 4 Distribution of (a) matrix and (b) $\delta^{\prime}$-phases orientations across thickness of $7 \mathrm{~mm}$ sheet after standard thermal treatment. 


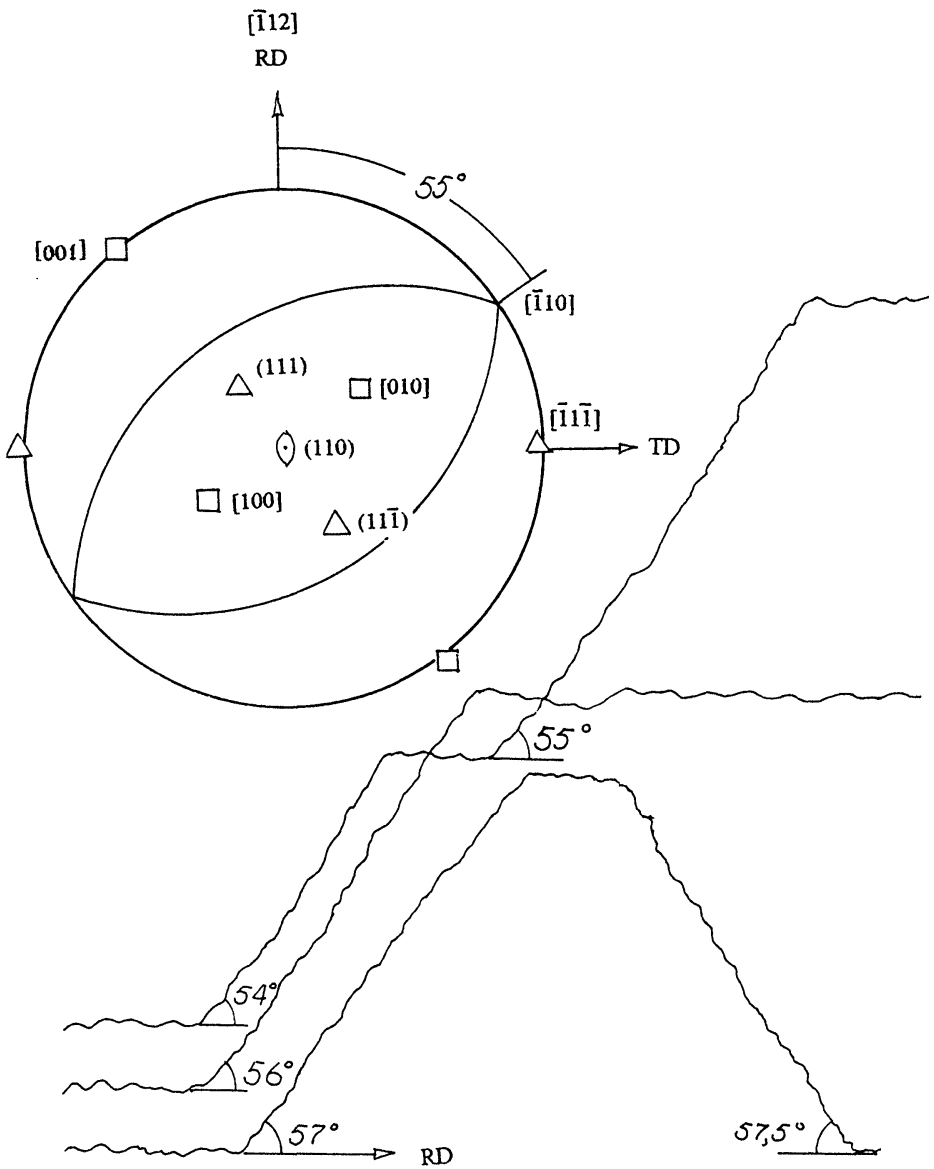

FIGURE 5 Stereographic projection of primary octahedral plane slip traces on (110) rolling plane in material with the mono-orientation (110)[112]. In the lower part propagation paths of fatigue cracks in several $5 \mathrm{~mm}$ center-notched specimens from central part of the $25 \mathrm{~mm}$ plate are shown.

the load axis directed either along the rolling $[\overline{1} 12]$ or the transverse $[\overline{1} 1 \overline{1}]$ directions. It is seen that the angles between rolling and transverse directions and the primary slip-plane trace on the rolling plane are $55^{\circ}$ and $35^{\circ}$ respectively. In the lower part of Fig. 5 the fatigue-crack paths for several TL specimens taken from the central part of the $25 \mathrm{~mm}$ plate are shown schematically. Good agreement between the primary slip 
plane trace and the experimental and calculated values of crack deviation angle is observed.

\section{DISCUSSION}

The fact that the sections of the crack propagation path are arranged parallel to $\{111\}\langle 110\rangle$ as well as the observed transgranular faceted fracture morphology point to the crystallographic mode of crack extension. As is generally known, the crystallographic faceted cracking in f.c.c. metals extends by slip along equally stressed $\{111\}\langle 110\rangle$ slip systems ahead of the crack tip; it is further accepted that crack propagation behavior is related to the type and extent of the plastic relaxation zone around the crack tip (Neumann, 1974). Due to evidence of co-planar $\{111\}$-type crack growth during conventional mode I based testing the occurrence of mixed mode fracture has to be considered in this material. For instance contribution of mode III shear displacements should be taken into account (Rao and Ritchie, 1992).

In brass textured $\{110\}\langle 112\rangle$ sheet and plate the close packed $\{111\}$ plane pairs are equally inclined to the rolling plane (110). The arrangement of the (110) rolling plane and the two equally shear-stressed $\{111\}$ planes for two mirror-plane symmetric components of the $\{110\}\langle 112\rangle$ texture is shown schematically in Fig. 6. In the upper part of Fig. 6 the component with pair of octahedral planes (111) and (11) equally inclined to the (110) plane is shown. The value of the intersection angle of $70^{\circ}$ between the (111) and (11) planes corresponds to the observed dihedral angle on the fracture surface of the TL specimens taken from the central part of the $25 \mathrm{~mm}$ plate. The traces of the (111) and (111) planes on the rolling plane are tilted by $55^{\circ}$ to the rolling direction. As pointed out above, the deflection of the crack propagation direction in the TL center-notched specimens from the principal direction has the same average value of $55^{\circ}$.

In the lower part of Fig. 6 the component which is mirror-plane symmetric with respect to the one discussed above is presented. As can be seen the traces of the (111) and (11) planes from the first and second components are also intersected at an angle of $70^{\circ}$. This value corresponds to the angle observed at the zig-zag line which formed when the propagating crack path in the TL specimens changed their direction to 


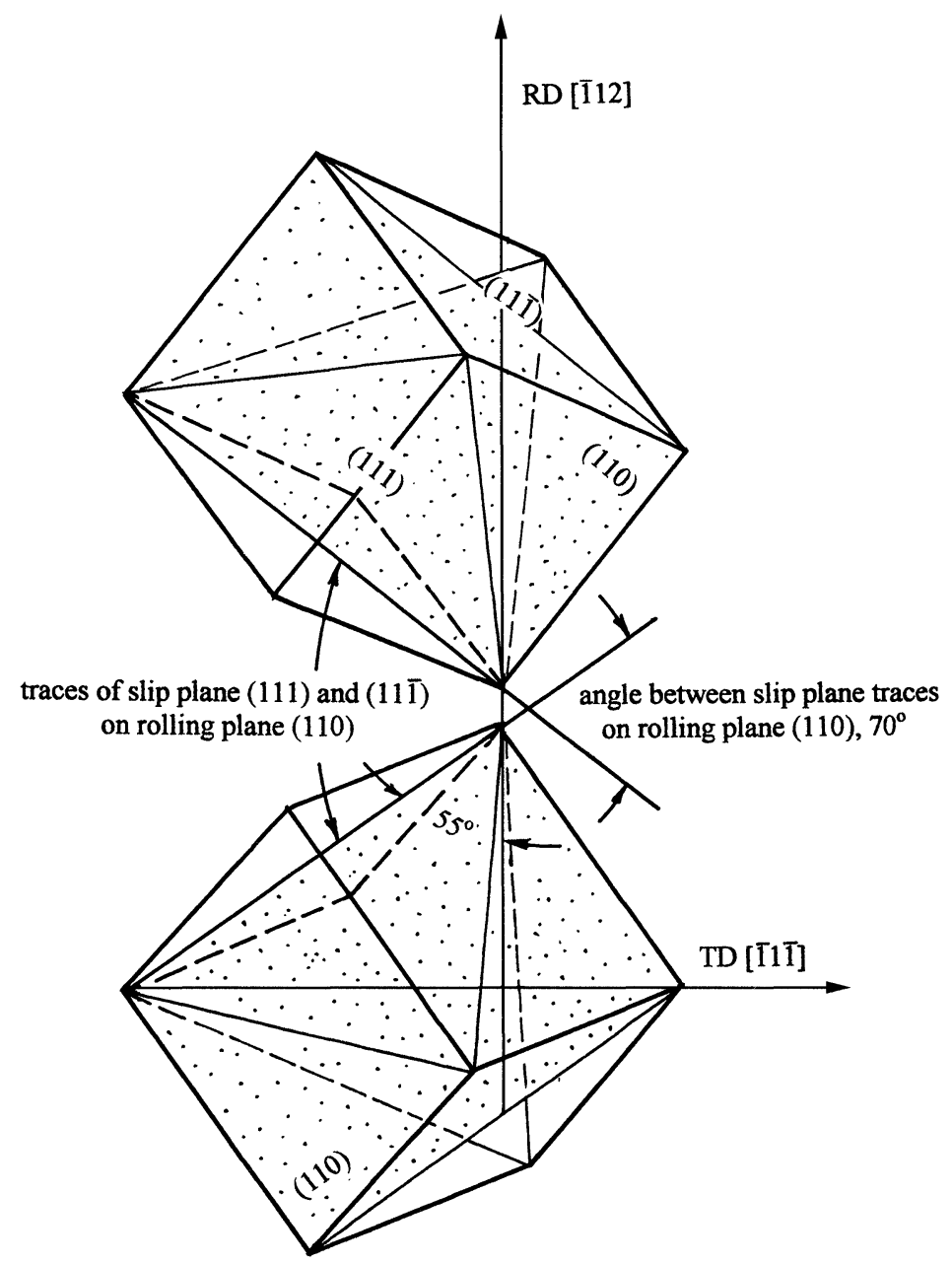

FIGURE 6 Illustration of relative orientations (111) and (111) primary slip plane traces on rolling plane (110) for $\{110\}\langle 112\rangle$ textured f.c.c. material with mirror-plane symmetry oriented grains (RD and TD: rolling and long transverse directions).

the mirror-plane symmetric one. Such a crystallographic extended crack path change may arise from the occurrence of several groups of adjacent grains with mirror-plane symmetric orientation relative to each other. A fatigue crack path extending through the regions of adjacent grains intersects the boundaries that divide differently oriented areas. As a 
result, the crack propagation direction is changed to the mirror-plane symmetric one. This conclusion is confirmed by the fact that the pole figure of a fracture surface consisted of the two mirror-plane symmetric components (100)[011] and (221)[114]] (Fig. 3). The macroscopic plane of the fracture surface aligns along a $\{100\}$ plane as usually observed in f.c.c. metals.

As mentioned above the formation of highly tortuous and deflected crack paths in $\mathrm{Al}-\mathrm{Li}$ alloys is stimulated by the presence of shearable $\delta^{\prime}$-precipitates (Rao and Ritchie, 1992; Wu et al., 1994). In the first stage of deformation involving alternating shear on two equally stressed slip systems the active dislocations shear the $\delta^{\prime}$-particles and form antiphase domains. Thus, trailing dislocations move on the same slip plane at a lower stress to restore the original order. The resulting motion of superdislocation pairs gives rise to a particularly inhomogeneous planar deformation mode localized along planar slip bands parallel to the $\{111\}$ slip planes. During later stages of deformation cracking occurs along these slip bands and microcracks, localized at the sheared $\delta^{\prime}$-precipitates, coalesce together to form the observed rough crystallographic faceted fracture surface.

The distinction in mixed mode crack behavior in LT and TL centernotched $5 \mathrm{~mm}$ specimens which were cut from the $25 \mathrm{~mm}$ plate could be related to the misorientation of matrix and $\delta^{\prime}$-phases and hence to the variation in the contribution of mode I and II fracture. For $\{110\}\langle 112\rangle$ textured matrix phase and $\{100\}\langle 001\rangle$ oriented $\delta^{\prime}$-phase in LT and TL specimens the values of the Schmid factor $|m|$ were estimated by calculating the resolved shear stress on the active octahedral slip planes in plane stress condition. This estimation indicates that in the LT specimen the Schmid factors for $\delta^{\prime}$ and matrix phase are equal, while in the TL specimen the $m$ value for the $\delta^{\prime}$-phase is larger by $14 \%$ which corresponds to a geometric softening of the $\delta^{\prime}$-phase in the TL specimens. The above effect along with work softening through the disordering and dissolving of $\delta^{\prime}$-particles by repeated dislocation movement resulted in particularly inhomogeneous planar deformation mode.

It seems reasonable to state that the observed variation in $\delta^{\prime}$-phase orientation is defined by the formation mechanism of the $\delta^{\prime}$-particles, which are usually considered to be coherent with the matrix. During spinodal decomposition of $\mathrm{Al}-\mathrm{Li}$ solid solutions the ordered domains inherit the matrix texture, and furthermore, the $\delta^{\prime}$-phase tends to remain 
coherent with the matrix up to large particle diameters (Radmilovič et al., 1989). Accordingly, the different texture of the $\delta^{\prime}$-phase (Fig. 4(b)) indicates that the $\delta^{\prime}$-particles form by a nucleation and growth mechanism. Under such circumstances, a preferable growth of second phase particles defined by minimum surface energy depending on the orientation of the precipitates relative to the matrix can account for the observed texture variation.

In the $5 \mathrm{~mm}$ specimens from the central part of the $25 \mathrm{~mm}$ plate a distinct crystallographic mode of fracture is stimulated by the strong $\{110\}\langle 112\rangle$ texture. However, the texture in compact specimens of the $25 \mathrm{~mm}$ plate is inhomogeneous across the thickness. Whereas in the central part the $\{110\}\langle 112\rangle$ texture developed, in the surface regions the shear texture (001)[110] dominates. This texture inhomogeneity will hamper the development of local planar deformation through the thickness, which is a characteristic feature of the $5 \mathrm{~mm}$ thick specimens with a homogeneous texture. During extention of slip deformation through the boundaries that divide different layers with different texture types, stress concentrations will arise and, as a consequence, delamination of the material is developed (Roven et al., 1990; Rao and Ritchie, 1992). Hence, the delamination observed in the present material on the fracture surface of compact specimens of the $25 \mathrm{~mm}$ plate could be the result of such through-thickness texture variation.

In the center-notched specimens taken from the $7 \mathrm{~mm}$ sheet with inhomogeneous texture across the thickness the crystallographic mode of fracture is non-dominant and contribution of mode I fracture is essential. As in the above case, the variation of texture across the sheet thickness results in material delamination and also in cavern formation.

\section{CONCLUSION}

1. The matrix and $\delta^{\prime}$-particle textures in 1,2 and $7 \mathrm{~mm}$ sheets and $25 \mathrm{~mm}$ plate of the 1441 alloy have been studied. The semiproduct textures are inhomogeneous through the thickness. The texture component distribution and the texture type strongly depend on the rolling operation.

2. The matrix shear texture $\{100\}\langle 011\rangle$ penetrates up to 0.25 sheet thickness. With an increase in the degree of deformation the 
$\{110\}\langle 112\rangle$ plane deformation texture (the brass type) transforms into the $\{123\}\langle 541\rangle$ texture.

3. The $\delta^{\prime}$-particle orientations in the sheets correspond to the matrix texture components. However, the pole densities in the first case are substantially lower. In bulk semiproducts the $\delta^{\prime}$-precipitates have, at the same time, the cubic orientations.

4. The $\{110\}\langle 112\rangle$ matrix texture stimulates the crystallographic material fracture during fatigue crack propagation testing. The type of fracture strongly depends on the matrix texture homogeneity and the $\delta^{\prime}$-particles orientations.

\section{References}

Fridlyander, I.N., Shamray, V.F., Babareko, A.A., Setjucov, O.A. and Egis, I.V. (1994). Crystallographic anisotropy of yield stress in aluminium-lithium alloys semi-products. Doklady Akademy Nauk, 336, 348-351.

Neuman, P. (1974). New experiments concerning the slip processed at a propagating fatigue fracture - I. Geometry of slip processes at propagating fatigue crack - II. Acta metallurgica, 22, 1155-1178.

Radmilovič, V., Fox, A.G. and Thomas, G. (1989). Spinodal decomposition of Al-rich alloys. Acta metallurgica, 37(9), 285-294.

Rao Venkateswara, K.T. and Ritchie, R.O. (1992). Fatigue of aluminium-lithium alloys. International Materials Reviews, 37, 153-185.

Roven, H.J., Starke, E.A. and Hjelen, J. (1990). Effect of texture on delamination behavior of A 8090-Type Al- $\mathrm{Li}$ alloys at cryogenic and room temperature. Scripta metallurgica et materialia, 24, 421-426.

Shamray, V.F., Babareko, A.A. and Setjucov, O.A. (1997). Crystal geometry of fracture surface and fracture mechanisms identification in textured large grain $\mathrm{Al}-\mathrm{Li}$ alloys. National Conference on Application of X-ray, S-ray, Neutrons and Electrons for Materials Investigation, 25-29 May, 1997, Moscow, Dubna.

Wu, X.J., Walace, W., Raizenne, M.D. and Koul, A.K. (1994). The orientation dependence of fatigue crack growth in $8090-\mathrm{Al}-\mathrm{Li}$ plate. Metallurgical and Materials Transaction A, 25, 575-587.

Zeng, H.X., Ahmad, M. and Engler, O. (1994). Mat. Sci. Technol., 10, 581-591. 